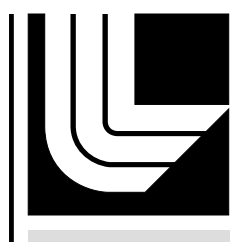

LAWRENCE LIVERMORE N A TIO NAL LABORATORY

Computers At Work On Ultrafast Laser Design

C. V. Filip

January 18, 2012

Optics And Photonics News 
This document was prepared as an account of work sponsored by an agency of the United States government. Neither the United States government nor Lawrence Livermore National Security, LLC, nor any of their employees makes any warranty, expressed or implied, or assumes any legal liability or responsibility for the accuracy, completeness, or usefulness of any information, apparatus, product, or process disclosed, or represents that its use would not infringe privately owned rights. Reference herein to any specific commercial product, process, or service by trade name, trademark, manufacturer, or otherwise does not necessarily constitute or imply its endorsement, recommendation, or favoring by the United States government or Lawrence Livermore National Security, LLC. The views and opinions of authors expressed herein do not necessarily state or reflect those of the United States government or Lawrence Livermore National Security, LLC, and shall not be used for advertising or product endorsement purposes. 


\section{COMPUTERS AT WORK ON ULTRAFAST LASER DESIGN}

Abstract: Ultrafast lasers stand up to their name. They have had such a rapid ascent in the last decade that optical engineers did not get a fair chance to instill much computer-aided design into them. But there are many incentives to catch up with the computerized technology.

Catalin Filip

Lawrence Livermore National Laboratory

\section{Ultrafast Lasers Foreword}

Power and speed: these are two qualities in big demand not only in sports or sports cars, but also in the world of lasers. And although that seems to be crystal clear to us today, it may not have been the case in the early beginnings of the laser. Picture yourself glancing over Theodore Maiman’s shoulder in 1960 and saying: “Don’t worry about the power, these things will become more than a million million times more powerful in the next 50 years. Really, don't look at me like that, it's true."

Of course, that is possible by simultaneously increasing the pulse energy and reducing the pulse duration. Ultrashort pulses have durations from picoseconds (ps) down to femtoseconds (fs). In the space domain that amounts to pulses from a millimeter to less than 10 micron thick which makes these pulses look like very thin coins or pancakes. As mystifying as it looks, it is possible to produce such pulses in laser cavities that are much longer than that. The phenomenon responsible for such achievement is called mode-locking and the laser is called an ultrafast oscillator. The necessary ingredients are a gain medium with a wide bandwidth and an intracavity mode-locking element. Although dye lasers were the first to be mode-locked, it was the solid-state and later the fiber lasers that truly cemented the name and released the potential of ultrafast lasers.

The output of an oscillator is a continuous train of pulses, typically with up to $1 \mathrm{~W}$ of average power. That is a mere percentage compared to what a high-power cw lasers can deliver. However, the appeal of ultrafast oscillators stems from its short pulses. Biomedical applications such as multiphoton imaging rely heavily on their peak power to produce large fluorescence signals while leaving the specimen with minimal damage due to absorption and heating effects. On the other hand, the micro-machining field relies on absorption and heating. The neat cuts and welds that are made with ultrafast lasers are better than those made with longer pulses or with traditional arc or plasma welding machines. That is simply because the laser pulse is over before the molten material starts boiling and spilling over.

For welding and cutting some materials though, the approximately $10 \mathrm{~nJ}$ of energy in each pulse produced by an ultrafast oscillator is too small. More energy is needed, for example, to write a variety of patterns such as gratings within transparent, less absorptive media or for the generation of attosecond pulses. Direct amplification of ps and fs pulses proved to be disastrous, literally. The electric field of directly amplified pulses is intense enough to change the structure of the material it propagates through. That in turn feeds back onto the phase of the laser pulse, a phenomenon called self-phase modulation that in the end results in optical damage of the 
material. This seemed to be a show stopper in the 60s and 70s, but laser scientists managed to find a way around direct amplification in 1985.

\section{Enter The CPA}

A simple idea was demonstrated by Donna Strickland and Gerard Mourou in their 1985 Optics Communications paper. They started with a picosecond oscillator pulse that was purposely stretched in time in an optical fiber to reduce its peak power. The 300 ps stretched pulse was further amplified in a laser amplifier and then compressed in a standard two-grating compressor to produce 2 ps pulses with $1 \mathrm{~mJ}$ of energy. The technique was dubbed chirp-pulse amplification, or simply, CPA. Since then, significant technological progress, such as the introduction of the Titanium-Sappire (Ti:S) material, brought the pulse duration down to less than $10 \mathrm{fs}$ and the energy up to tens or even hundreds of Joules. Record levels of peak power (1.1 Petawatt) were achieved in 1999 at Lawrence Livermore National Laboratory and many plans are being developed around the world to increase it to beyond 100 Petawatt as it is the case with the Extreme Light Infrastucture (ELI) project. The CPA technique has evolved and refined constantly since 1985 and the results have been impressive.

\section{Dispersion In Ultrafast Lasers}

The most important thing in the design of a CPA system is how the pulse is being stretched, amplified, and then subsequently compressed. In order to produce high energy, compressed pulses, the oscillator pulses need to be stretched as much as possible. The more stretching is produced, the more difficult it is to compress the amplified pulses back to their original width or near their transform-limited duration. The fidelity of the stretching-compression scheme becomes the very key element of the laser design. That is valid for all CPA systems, no matter the power, size or pulse duration.

Laser scientists showed extreme ingenuity over the years and constructed a variety of schemes to stretch/compress laser pulses. Very long optical fibers, due to their dispersive capabilities, were initially used as stretchers. Later, spatially dispersive optical elements based on diffraction gratings became widespread because they could stretch optical pulses much more than fibers. Overall, laser scientists stretched pulses quasi-linearly in time both in a positive (red colors first) and a negative (blue colors first) dispersion way using lenses, gratings, spherical mirrors, parabolic mirrors, etalons, etc. The compressor designs have also been very diverse from grating arrangements to bulk material and chirped mirrors.

With such a wide variety of dispersive elements and schemes, laser scientists began paying attention to the stretching ratio and the residual phase of the compressed pulse. The former is defined as the ratio between the duration of the stretched pulse and the duration of the oscillator pulse. This factor determines how much energy can be amplified. The latter is being used to show how good the compression process will be. The residual phase is also used in conjunction with the time-bandwidth product (TBP) to characterize the compressed pulse.

\section{Grating Stretchers And Compressors}

Among all the different dispersive elements used in CPA systems, the diffractive grating proved to be the most successful. Very large stretching ratios with good, compressed pulses were achieved. Historically, the compressor developed first, even before CPA. It was Treacy in 1969 
who showed how a two-grating assembly can compressed a positively chirped pulse. The stretcher was developed much later by Martinez in 1987 who realized that a telescope placed in between the gratings of a compressor will effectively reverse the dispersion sign. The simple but ingenious work by these scientists helped define the path for the modern ultrafast amplifier technology.

Today, almost any CPA system that produces more than $1 \mathrm{~mJ} /$ pulse, industrial or scientific, utilizes diffraction gratings in its design, both in the stretcher and the compressor. Initially, following Martinez's work, any stretcher design was considered the exact opposite of a compressor, including stretchers with lens telescopes. Later, experimental studies pointed out the limitations of refractive stretcher designs, due to aberrations and additional dispersion factors introduced by the glass. Reflective stretcher designs were quickly developed after that.

One such stretcher that is considered the standard for any commercial, $\mathrm{kHz}$ repetition rate Ti:S CPA laser has its telescope made of one spherical (or parabolic) mirror and another flat mirror. The stretching ratio is as high as 10,000 and the footprint is roughly $1 \mathrm{x} 4$ square feet. This stretcher proved to be much better than a lens-telescope stretcher and it was successfully used to amplify 40 fs pulses. Below 40fs though, the dispersion characteristics of the stretcher need to be calculated very precisely because even its smallest aberrations become important.

But how do we accurately calculate the dispersion of a relatively complex three-dimensional optical system where the rays bounce back and forth many times in between its components? The obvious, simple, and most accurate way is to utilize the help of a commercial, well-tested ray-tracing program such as Zemax, CodeV, Oslo, Fred, etc. This method is vastly superior to previously used analytical calculations and two-dimensional ray-tracing computations. The optical path is calculated for many wavelengths within the wavelength interval of interest. This needs to be done for the stretcher, amplifier material and for the compressor. The stretcher is the hardest to calculate because of the multiple passes in between its components. The dispersion of the amplifier material is easy to compute because the laser is usually made of extremely well characterized glasses, sapphires or other crystals. The compressor is easy to study too because exact analytical formulas have been worked out. The compressor is, in fact, a fantastic resource for anybody who wants to benchmark a ray-tracing program in order to avoid potential errors.

There are important benefits when analyzing the dispersion of a CPA system using a 3D computer model. Let's closely look at a special case of a CPA system, the optical parametric chirped pulse amplifier (OPCPA). In an OPCPA the laser pulse is amplified in a nonlinear crystal by as much as 10,000 to 100,000 times. Two or more crystals can be used sequentially to further increase the amplification. Since these crystals are generally thin, there is not much dispersion introduced by them. Therefore, the designer of the OPCPA just needs to make sure the compressor is compensated by the stretcher exactly, or, in other words, the stretcher is an exact opposite of the compressor. And since all stretchers are not alike, the 3D computer modeling is needed to pinpoint which one is the most appropriate for the task. For the abovementioned OPCPA case, studies showed that, an Offner-type stretcher made of two spherical mirrors, one concave and one convex, will be the best match for the compressor.

There are other CPA designs where significant dispersion is introduced by the amplifying material, as it is the case of a regenerative amplifier with intra-cavity polarizers, Pockels cells, windows, etc. The total length of the material could add up to 1-2 meters and in this case a full 
analysis including the stretcher, compressor and the amplifier is necessary. Any combination of grating groove densities, curvatures, distances and incidence angles, both in the stretcher and the compressor, could result in the best dispersion compensation case. What ultimately is important is the precise calculation of the dispersion characteristics of all the components of the CPA laser system and that can only be achieved by using a 3D computer model.

\section{Misalignment In CPA Lasers}

And there are even more benefits from modeling the components of a CPA laser. One of them is related to misalignment that develops in all lasers, to some extent. The typical causes are: mechanical stress, temperature variations, vibrations, shocks and chemical instability of the adhesives that are used to glue the optics in their mounts. The CPA systems are more sensitive to misalignment because of their particular relationship between beam pointing and dispersion. Within the CPA class of lasers, the most sensitive are the free-space CPA systems, all fiber lasers being less sensitive to misalignment.

For a free-space CPA laser, variations in the beam pointing in the stretcher and the compressor will affect the dispersion balance of the laser. That, in turn, changes the pulse duration. It is possible that some components present within the stretcher and the compressor will become misaligned too, and that will affect the pulse duration as well. Dispersion-wise, some of these components are more "important" than others and sometimes misalignment in one direction does more damage than misalignment in the other. Figuring out what are the critical optics in a CPA system is very important for industrial systems that strive for extreme beam pointing and pulse duration stability under adverse environmental conditions.

Misalignment can be a big problem for a scientific research laser too. Let's have a closer look at a potential case. Let's consider a high-end laser such as a 100 terawatt, TitaniumSapphire CPA system generating sub-30 fs pulses in a $50 \mathrm{~mm}$ beam. The compressed pulse is focused onto the target by an off-axis parabola (OAP). We assume a two-grating compressor is used and the compressor is aligned perfectly in air. A diffraction-limited spot size near $5 \mu \mathrm{m}$ can be achieved. However, the experiment is performed in vacuum. Therefore, small bending and pulling of the entire vacuum chamber will determine minuscule movements of the compressor optics. If the $2^{\text {nd }}$ grating rotates in the plane of dispersion by only 0.05 degrees, less than $1 \mathrm{mrad}$, the "in-air", otherwise diffraction-limited spot will transform into an aberrated spot. The beam pointing will change as well as the dispersion balance, pulse duration, spot size, and ultimately the intensity in the focal spot. By utilizing a ray-tracing program, the laser engineer can calculate the magnitude of these changes and, based on the results, recommend solutions, possibly a stiffer (and of course more expensive) mechanical design of the vacuum chamber. Note that the mechanical behavior of the optic holders in a vacuum chamber can be predicted quite accurately with commercially available finite element analysis (FEA) software packages. Other potential solutions may be studied with the ray-tracing program, such as compensating with the vertical retro-reflector and/or the OAP or maybe by changing the beam pointing into the compressor. Then, the dispersion compensation needs to be checked again. A simple computer program could therefore be very helpful to a million-dollar laser. 


\section{Stray Light, Some Want It, Some Don't}

The scattered light or the stray light is present in any laser but is mostly dangerous in highpower systems. This light is generated by all the optics in the laser to some extent and follows beam paths other than those for which the laser was designed. For example, lenses are known for producing "back reflections" from their surfaces. Although the reflections are very weak, if the stray light ends up focused on the surface of another optic in the laser, it could potentially damage it. Computer models built in the non-sequential ray-tracing mode are (again!) extremely useful in determining the most appropriate curvatures and positions of lenses and telescopes. Sometimes, the simple solution is to rotate a lens to avoid damaging other optics. For a high-end ultrafast laser, however, one has to use a ray-tracing program to estimate what are the implications of such a small angle rotation for the final spot size and pulse duration.

The stray light can be a saint and a demon at the same time. On the one hand, many simple, mostly industrial CPA lasers are being used for tasks that do not require compressed pulses with very high temporal contrast. The stray light that bounces off the stretcher and compressor gratings at almost any angle can then be visualized with an infrared viewer. That allows for a fast and easy alignment check. On the other hand, for cutting edge scientific systems, the stray light poses a grand challenge and possibly a fundamental limitation. Mostly that has to do with the light that is scattered from the compressor grating surfaces. The mechanism is simple: the uncompressed pulse hits the $1^{\text {st }}$ grating and a very tiny part of it will scatter at angles and directions other than the design ones. A similar process is reproduced on the $2^{\text {nd }}$ grating and then again on the subsequent hits after the pulse is being sent back by the vertical retro reflector. Because the scattered light does not follow a compressible path, it will end up at the intended target either earlier or later than the compressed pulse, thus contributing to the generation of a temporal pedestal. The spatial contrast worsens too because part of the scattered light will travel as slightly different angles than the "compressed" beam and it will be focused around the final spot. No matter how "clean" the uncompressed pulse is, some temporal and spatial background will be generated by the stray light in the compressor. The amount of energy contained solely in the stray light that ends onto the target could be larger than $0.01 \%$ of the incident energy. If the laser output is, for example, near $100 \mathrm{~J}$, the stray light could contain as much as $10 \mathrm{~mJ}$. If we assume that half of this light will reach the target before the main pulse and half after, that is still more than enough energy to vaporize the target material and create a, what is called in the scientific language, pre-plasma. The consequence is, simply, that the experiment performed this way is different than what was intended and no clear scientific conclusion can be reached.

Clearly one solution to this problem is to manufacture gratings that scatter less light. Good progress has been made with holographic gratings showing great improvement over the ruled ones. Multi-layer dielectric coatings appear to scatter less than bare gold coatings. The scattering angle and characteristics also depend on the groove density and substrate material as well as the manufacturing process. How would a laser engineer proceed about taking into account all these options and finding the best solution? Part of this work would be to first characterize the scattering in a small experimental setup. Then the resulting data can be entered into the raytracing program that can analyze various compressor designs without actually having to build one. In the end, the best design will prevail. 


\section{From the computer screen into production}

The first lasers were basically formed of two mirrors, a flash lamp and the gain medium. Now, roughly 50 years later, ultrafast lasers are becoming more and more complex. Many more parts and optics are being used to build one. Every single component inside an ultrafast laser needs to perform as designed and expected. If one fails, then the entire laser fails. There is no margin for error, no mercy. This concept was well understood in the mid 60s when the first mechanical computer-aided design (CAD) programs were built for automotive and aerospace companies such as General Motors and Lockheed Martin. Sure, we cannot compare the laser industry with the two above-mentioned giant industries. But more ground needs to be gained in the optical simulation territory because of some benefits such as the ones showed here for ultrafast lasers, accurate dispersion calculations, misalignment studies and stray light analysis.

Progress is being made, the optical CAD programs are now being integrated with the mechanical ones together with finite element analysis (FEA) capabilities. More and more complex photonics problems are being tackled. Laser beams and resonators are being simulated in environments dominated by thermal effects. Some of the traditional CAD programs have now the capability to trace rays and work with free-form or user-defined optical surfaces. For now, data is quickly exchanged between optical, mechanical and analysis modules. Fully-integrated codes are currently being developed. Once available, they will help the optical engineers find the best design, save time and money and avoid errors. Some of the laboratories where prototype systems are being tested before entering the manufacturing process will be replaced by a wall full of computers. Hopefully, a decade from now, some lasers will go directly from the computer screen into production.

This work was performed under the auspices of the US Department of Energy by Lawrence Livermore National Laboratory under contract DE-AC52-07NA27344.

\section{References and Resources}

- D. Strickland and G. Mourou Opt. Commun. 56, 219 (1985).

- M. D. Perry et al Opt. Lett 24, 160 (1999).

- The extreme Light Infrastructure: http://www.extreme-light-infrastructure.eu/

- E.B. Treacy, IEEE J. Quant. Electron. QE-5 454 (1969).

- O.E. Martinez, IEEE J. Quant. Electron. QE-23 59 (1987).

- A. Offner, U. S. Patent 3748015 (1971). 
GRATING-BASED OPTICAL STRETCHERS

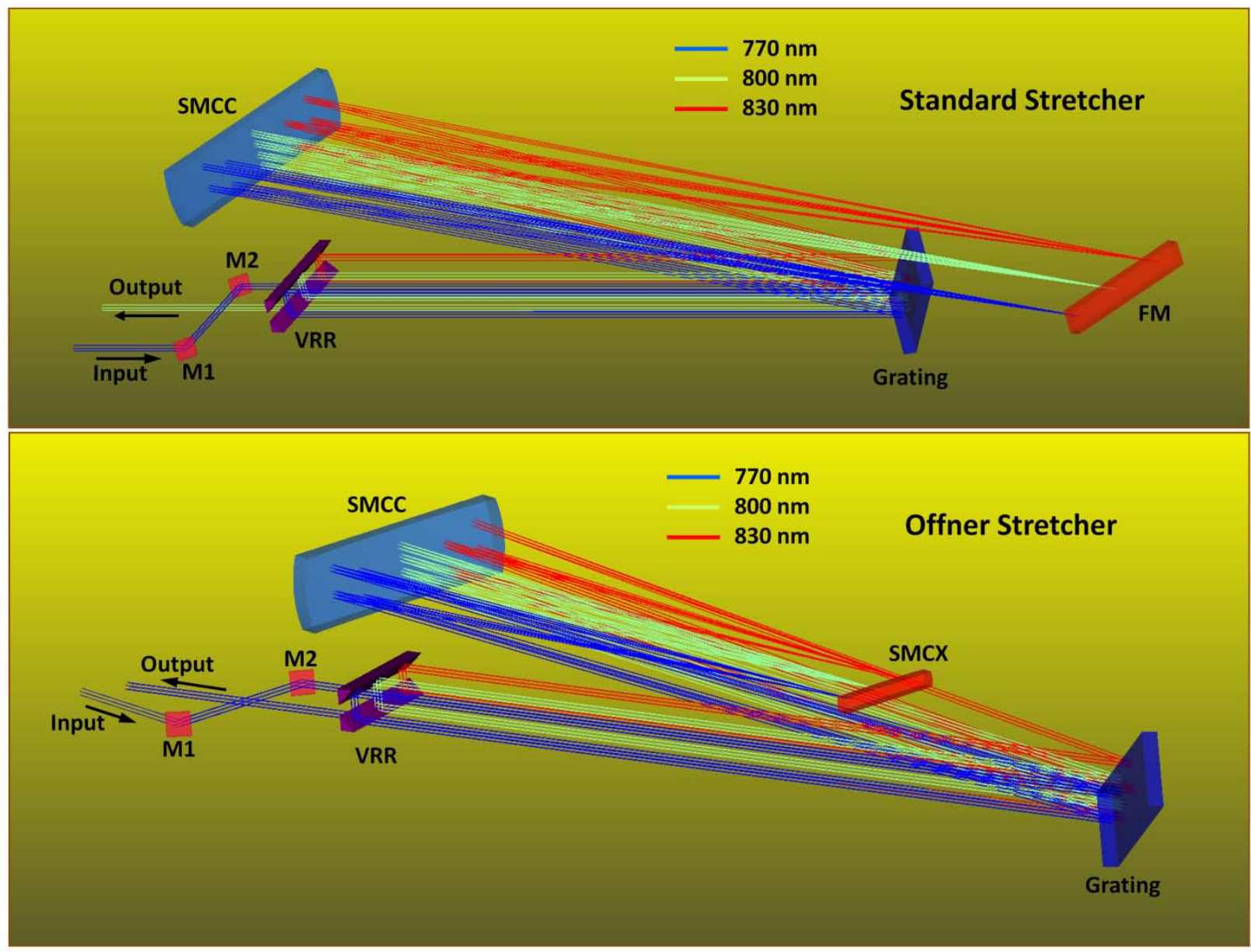

Fig. 1. Optical 3-D (there-dimensional) layout in Zemax showing the components of two optical stretchers and the beam propagation. M1, M2 and FM are folding mirrors, SMCC is a concave spherical (or parabolic) mirror, SMCX is a convex spherical mirror, VRR is a vertical retro reflector. 


\section{STANDARD GRATING COMPRESSOR}

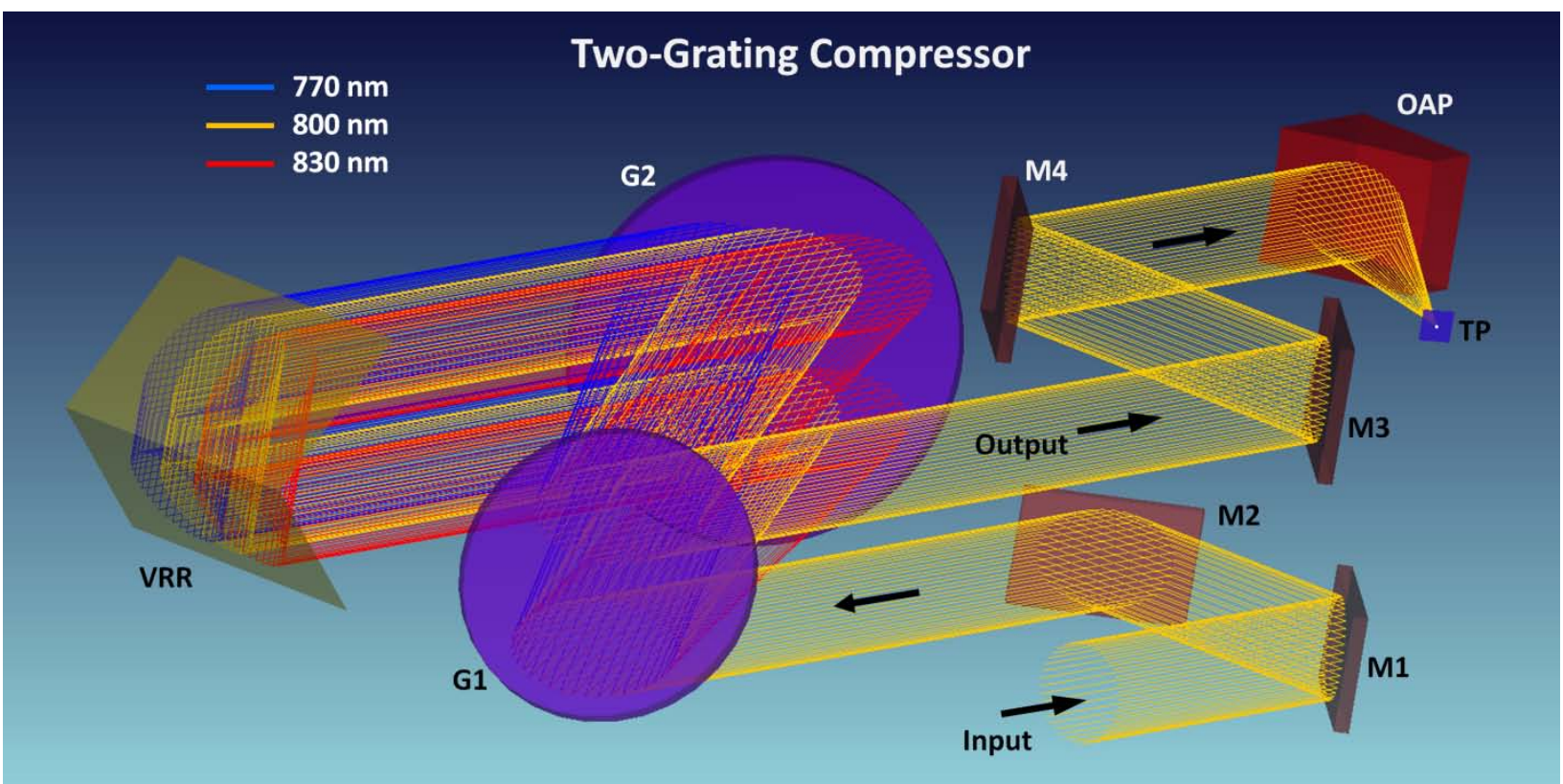

Fig. 2. Zemax 3-D (there-dimensional) layout of a compressor together with the off-axis parabola that is used to focus the $50 \mathrm{~mm}$ beam. M1 to M4 are folding mirrors, G1 and G2 are the diffraction gratings, VRR is the vertical retro reflector, OAP is the off-axis parabola, TP is the plane where the beam focuses and a target is placed. 


\section{DISPERSION CALCULATION RESULTS}
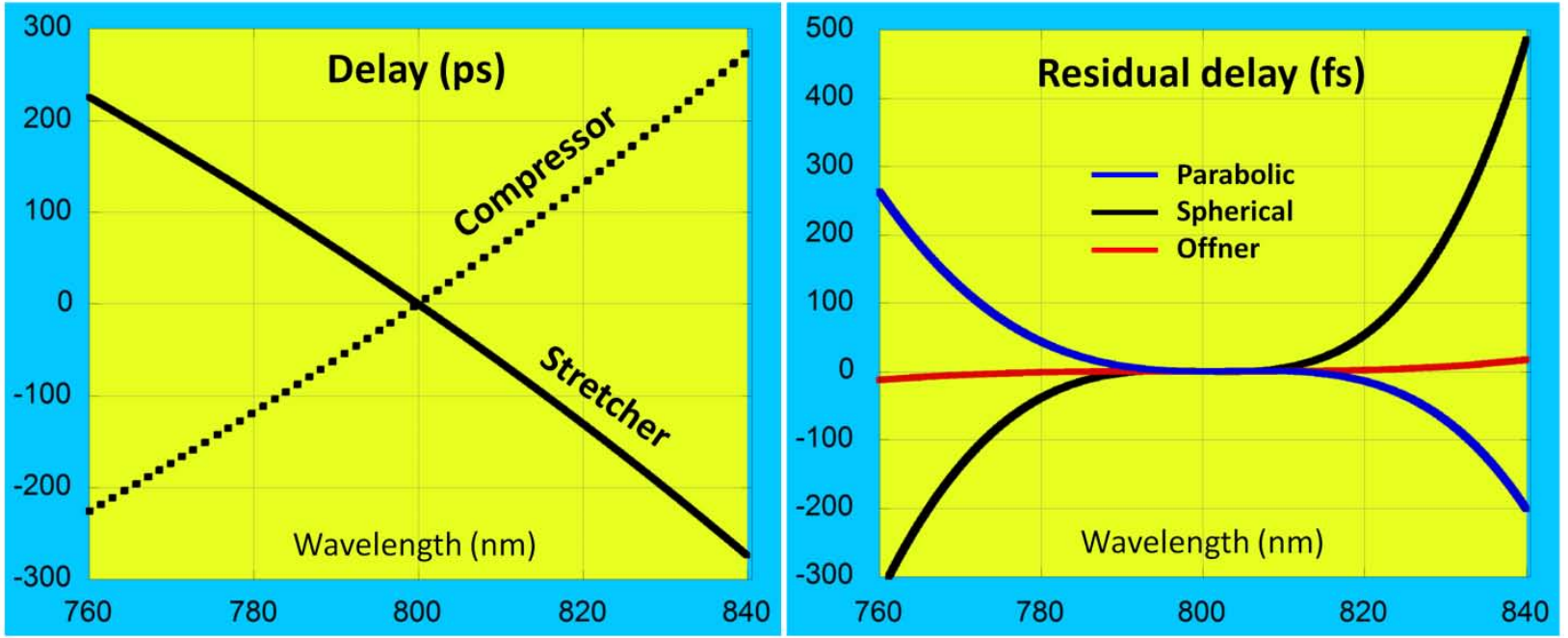

Fig. 3. (Left) Group delay introduced by a compressor and its counterpart stretcher (same grating, incidence angle and effective length). (Right) The residual delay for three different stretcher types obtained by summing the two curves from the left plot. The Offner stretcher is very close to being an exact opposite of the compressor. 


\section{COMPRESSOR ALIGNMENT ISSUES}
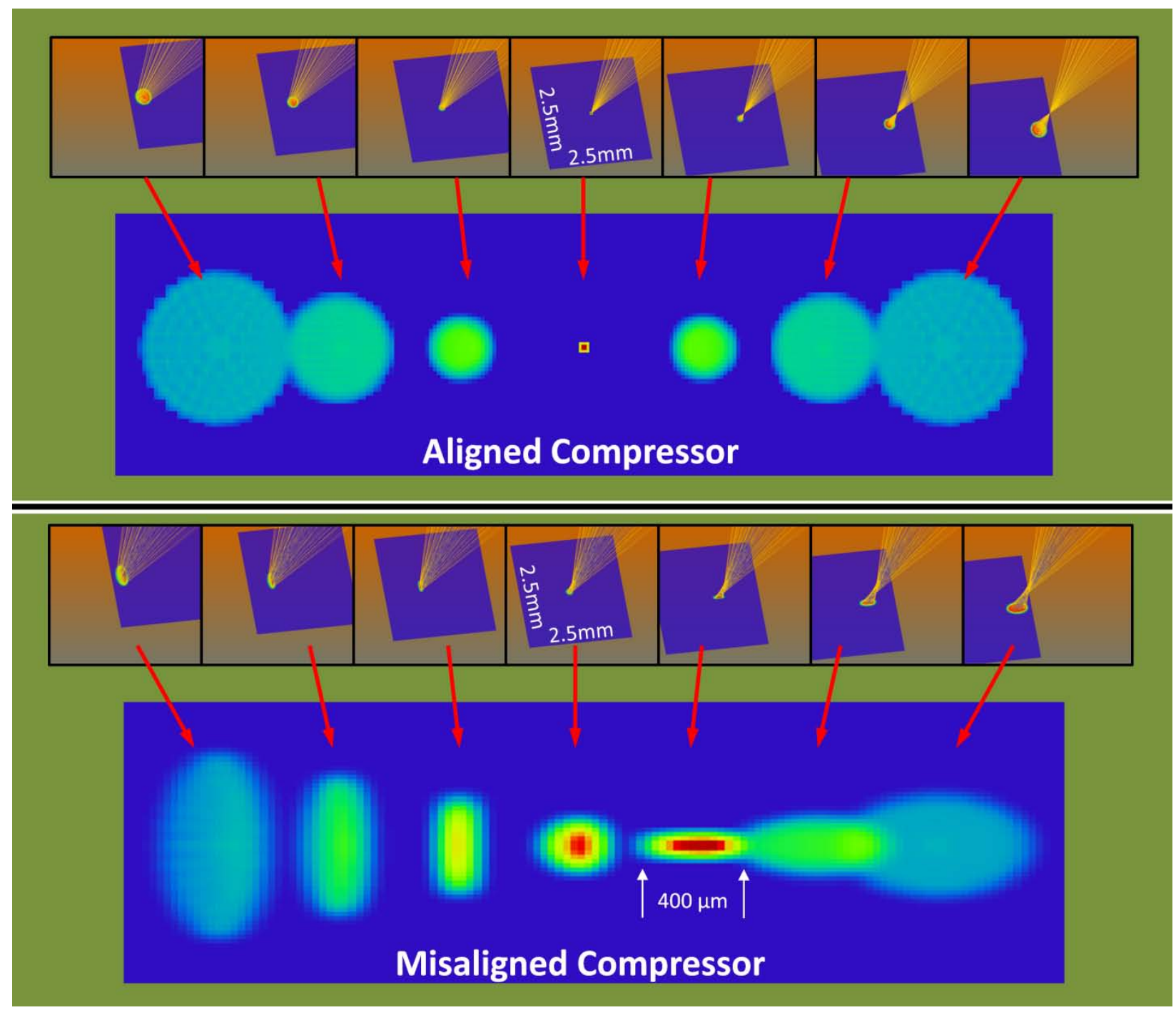

Fig. 4. (Top) The final focus images of a Titanium-Sapphire ultrafast laser done with Zemax ray tracing. A $2.5 \mathrm{~mm}^{2}$ square detector screen is scanned across the focus produced by an F/3.6 off-axis parabola. The compressor is perfectly aligned. The spot is diffraction-limited and pictured in the middle as a dot. (Bottom) Similar images if the compressor is misaligned by a 0.05 degree rotation of the $2^{\text {nd }}$ grating in the plane of dispersion. 


\section{COMPRESSOR-GENERATED STRAY LIGHT}

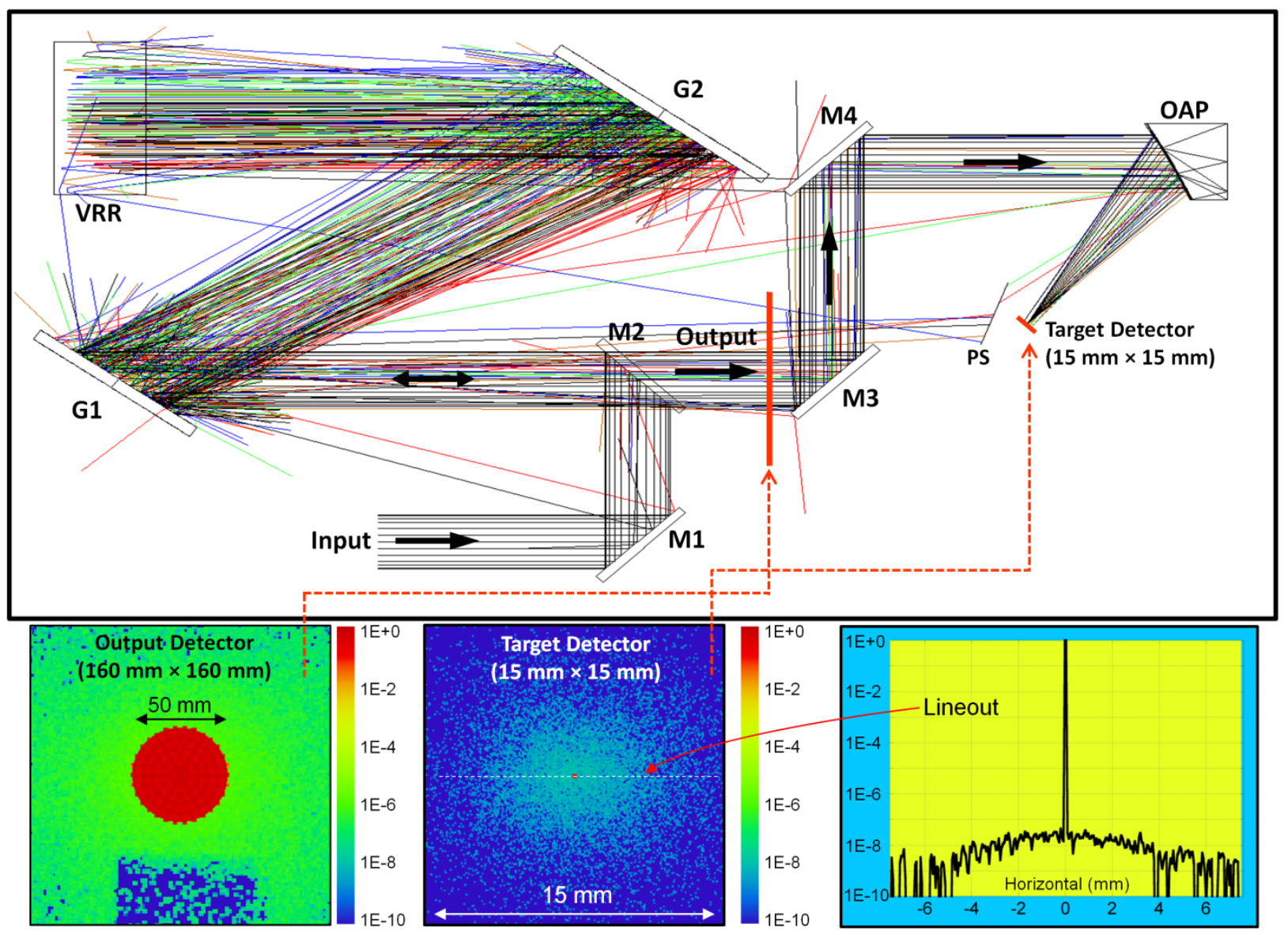

Fig. 5. (Top) Top view of the two-grating compressor together with the rays that form the normal beam as well as some of the stray light. The model assumes scattering on the gratings only at the level of a polished glass surface (very good gratings). Note that the output beam propagates above the folding mirror M2. PS is a protective screen that blocks the stray light coming from the left. (Left) The image recorded by a detector placed in the output beam. The shadow at the bottom of the image is given by M2. (Middle) The image recorded by a detector placed in the final focus or target plane. (Right) A lineout of the distribution of the stray light in the target plane. 